\title{
APOLLO_NG - a probabilistic interpretation of the APOLLO legacy for AVHRR heritage channels
}

\author{
L. Klüser, N. Killius, and G. Gesell \\ German Aerospace Center, German Remote Sensing Data Center, Oberpfaffenhofen, Weßling, Germany \\ Correspondence to: L. Klüser (lars.klueser@dlr.de)
}

Received: 19 March 2015 - Published in Atmos. Meas. Tech. Discuss.: 30 April 2015

Revised: 30 September 2015 - Accepted: 30 September 2015 - Published: 12 October 2015

\begin{abstract}
The cloud processing scheme APOLLO (AVHRR Processing scheme Over cLouds, Land and Ocean) has been in use for cloud detection and cloud property retrieval since the late 1980s. The physics of the APOLLO scheme still build the backbone of a range of cloud detection algorithms for AVHRR (Advanced Very High Resolution Radiometer) heritage instruments. The APOLLO_NG (APOLLO_NextGeneration) cloud processing scheme is a probabilistic interpretation of the original APOLLO method. It builds upon the physical principles that have served well in the original APOLLO scheme. Nevertheless, a couple of additional variables have been introduced in APOLLO_NG. Cloud detection is no longer performed as a binary yes/no decision based on these physical principles. It is rather expressed as cloud probability for each satellite pixel. Consequently, the outcome of the algorithm can be tuned from being sure to reliably identify clear pixels to conditions of reliably identifying definitely cloudy pixels, depending on the purpose. The probabilistic approach allows retrieving not only the cloud properties (optical depth, effective radius, cloud top temperature and cloud water path) but also their uncertainties. APOLLO_NG is designed as a standalone cloud retrieval method robust enough for operational near-realtime use and for application to large amounts of historical satellite data. The radiative transfer solution is approximated by the same two-stream approach which also had been used for the original APOLLO. This allows the algorithm to be applied to a wide range of sensors without the necessity of sensorspecific tuning. Moreover it allows for online calculation of the radiative transfer (i.e., within the retrieval algorithm) giving rise to a detailed probabilistic treatment of cloud variables. This study presents the algorithm for cloud detection and cloud property retrieval together with the physical prin-
\end{abstract}

ciples from the APOLLO legacy it is based on. Furthermore a couple of example results from NOAA-18 are presented.

\section{Introduction}

The cloud analysis tool APOLLO (AVHRR Processing Over cLouds, Land and Ocean) has been in use for more than 25 years now. It has been developed for cloud detection from Advanced Very High Resolution Radiometer (AVHRR) observations (Saunders and Kriebel, 1988). It also has been expanded (e.g., Gesell et al., 1989) and updated (Kriebel et al., 2003) on several occasions. The AVHRR cloud detection with APOLLO has been evaluated a couple of times (Kriebel et al., 2003; Meerkötter et al., 2004). Also in a wide range of other applications APOLLO has been used in adaptations to different satellite sensors. With observations of the (Advanced) Along-Track Scanning Radiometer ((A)ATSR) it has been used as cloud screening method for aerosol retrievals (Holzer-Popp et al., 2008) and with Spinning Enhanced Visible and InfraRed Imager (SEVIRI) observations for cloud screening as input to an infrared desert dust index (Klüser and Schepanski, 2009). Especially for the use with AATSR sensor-specific updates have been necessary (Holzer-Popp et al., 2008). APOLLO also has been used in dedicated experiments within the Aerosol_cci project of the European Space Agency (ESA). Moreover APOLLO input is used to compute surface solar irradiances (Oumbe et al., 2009; Qu et al., 2012), which is an important information for solar industry. It has furthermore been attempted to investigate the impact of desert dust aerosol on cloud properties from APOLLO data and collocated aerosol retrievals (Klüser and Holzer-Popp, 2010). 
During all these years of research with use of APOLLO a couple of limitations have been identified. Oumbe et al. (2013) propose to retrieve cloud effective radius together with the optical depth estimation in order to improve the modeling of cloud extinction for solar irradiance calculations. Klüser and Holzer-Popp (2010) reported that the lack of cloud effective radius retrieval is very disadvantageous in aerosol-cloud-interaction studies. Merchant et al. (2005) point out the advantage of a more flexible probabilistic cloud detection scheme. The same argument is brought by HolzerPopp et al. (2013) with a different focus: in comparing cloud masks from the aerosol_cci and the cloud_cci projects they found that about $20 \%$ of all observations were used neither for aerosol retrieval nor for cloud retrieval. The reason outlined in Holzer-Popp et al. (2013) was that aerosol retrievals require a cloud mask which is precise about clearsky pixels (a so called clear-conservative cloud mask). On the other hand, for retrieving cloud properties it is necessary to be sure that the pixel is cloudy (cloud-conservative cloud mask). Consequently, there is no single cloud mask fulfilling the need of both groups. Cloud detection has thus to be flexible with respect to the application it is used for.

Different versions of the APOLLO algorithm have evolved over time (e.g., Kriebel et al., 2003; Holzer-Popp et al., 2008; Klüser and Schepanski, 2009; Klüser and HolzerPopp, 2010). The evolution of varying versions for different purposes and sensors made it desirable to harmonize the sensor dependent versions again. Necessary requirements like the introduction of a cloud droplet effective radius retrieval along with the optical depth estimation (Nakajima and King, 1990), the use of modern representations of ice cloud optical properties (Baum et al., 2014) and the requirement for more flexible cloud detection (Merchant et al., 2005; Holzer-Popp et al., 2013) consequently motivated the development of a new cloud detection and cloud retrieval scheme based on the physical principles of APOLLO.

As a direct consequence, a new probabilistic cloud detection scheme has been developed from the basis of the APOLLO physics. One major goal of the next generation method is to be applicable with any satellite sensor providing the traditional five AVHRR channels (centered approximately at $0.6,0.9,1.6 / 3.7,11$ and $12 \mu \mathrm{m}$ ). Consequently, the new scheme does not use additional channels (like those centered at 8.7 or $13.4 \mu \mathrm{m}$ ), which are available on instruments such as SEVIRI or the new Visible Infrared Imaging Radiometer Suite (VIIRS) onboard the recently launched Suomi satellite. The probabilistic scheme still relies on the same physical assumptions as the original APOLLO scheme. The scheme is specifically designed to be applied to the full AVHRR series, which lacks the information of additional channels. Consequently, we feel it is justified to still call the method APOLLO although it is not exactly an update of the existing algorithm but rather a new approach using the same physical ideas. Consequently, the scheme will be called APOLLO_NextGeneration (or APOLLO_NG throughout this article). The AVHRR channel terminology is used, i.e., channel numbering from 1 to 5 with channel 1 referring to a red channel centered at $0.6 \mu \mathrm{m}$ and channel 5 referring to an IR channel centered at $12 \mu \mathrm{m}$ (see Kriebel et al., 2003). We are fully aware that the scheme will not provide fully consistent results for different sensors due to the varying sensor characteristics of the AVHRR family and the differences in sensor design for other instruments. But at least it uses a similar mathematical framework for all sensors without introducing specific additional information from one channel or another which is not available from AVHRR. Results from different sensors will thus be at least comparable, although they are not absolutely consistent.

Section 2 introduces the APOLLO_NG cloud detection algorithm. Section 3 deals with the detection of snow and its discrimination from clouds while Sect. 4 describes the retrieval of physical cloud properties subsequent to the cloud detection. It is followed by some examples in Sect. 5, a discussion of the algorithms and corresponding results in Sect. 6 and a concluding summary in Sect. 7.

\section{Probabilistic cloud detection}

\subsection{Algorithm heritage}

The original APOLLO cloud detection is based on five consecutive threshold tests, for which the thresholds are determined dynamically from the analyzed scene.

The five tests include the infrared gross temperature test, the dynamical visible cloud test, the spatial coherence test, the reflectance ratio test and the brightness temperature difference test. The physical ideas behind the five APOLLO cloud tests are that cloud tops are cold, bright or inhomogeneous or a combination thereof. Moreover water clouds can be identified by their reflectance ratio between two solar channels (being close to 1) as well as by differential absorption at two wavelengths in the infrared window.

Two of these tests were run twice with updated information. The succession of the respective tests fed into a bitadding scheme. Any observed AVHRR pixel has been said to be cloud-contaminated or fully cloudy, if a sufficiently large number of bits are added in the second run (see Saunders and Kriebel, 1988; Kriebel et al., 2003).

The classical APOLLO cloud detection tests are also incorporated in more modern cloud detection schemes like the cloud masking for the Moderate Resolution Imaging Spectroradiometer (MODIS) as reviewed by Frey et al. (2008). As modern sensors provide many more channels than the AVHRR family, the traditional APOLLO tests are nevertheless only a subset of cloud detection tests in modern schemes such as from MODIS. The new APOLLO_NG cloud detection scheme is based on the same physical principles and thus channels and channel combinations. It is designed to be applicable to AVHRR. Consequently, only the classical 


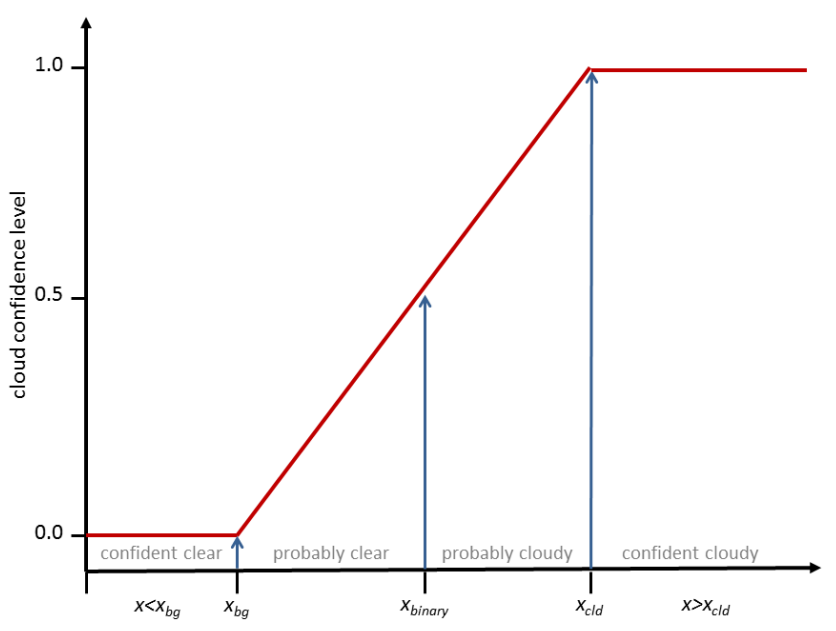

Figure 1. The linear approach of different confidence levels and thresholds used for the cloud probability estimation from an observation of variable $x$.

"AVHRR heritage" channels are used in APOLLO_NG. But, instead of binary yes/no information gained from "simple" threshold tests, the distance from the respective threshold is used as an estimate of the likelihood of cloud presence in the observation. A Bayesian probability update scheme then uses the cloud test probability (interpreted as confidence in observing a cloud with corresponding properties) for updating the overall cloud probability. Figure 1 showcases how the distance from the threshold can be interpreted as a cloud probability. Binary threshold methods such as the traditional APOLLO scheme assume that, if an observed value is greater (lower) than a threshold, it is "definitely" cloudy or cloud free. Thus it assigns a cloud probability of either 0 or 1 to a specific cloud test without allowing for fractional probabilities. Consequently, the probabilistic extension is more flexible (allowing all probabilities between 0 and 1) and is also stricter in the mathematical interpretation. It propagates the probability in a clearly prescribed statistical way with equal weight for all cloud tests to the final cloud probability.

After having obtained an estimate of the cloud probability, the cloud affected pixels (over land) undergo a snow detection test in the legacy of the Gesell et al. (1989) method. The minimum value of cloud probability for assigning an observation to the cloud mask can be set according to the user's needs. Consequently, the APOLLO_NG cloud detection can be tuned from clear sky confident (i.e., having low clear sky misclassification rate) to cloud confident (low cloud misclassification rate). This gives the user of the cloud detection scheme an increased cloud masking flexibility compared to the traditional APOLLO scheme.

Figure 2 shows a detailed flowchart of the APOLLO_NG cloud detection. In contrast to the traditional APOLLO scheme the information of each individual cloud test feeds as $P_{\text {cld }}$ into the outcome of the subsequent test. The
APOLLO_NG cloud detection scheme should be seen as a new algorithm rather than an update of the traditional APOLLO method. Nevertheless, both are based on the same physical principles of cloud detection.

\subsection{Cloud probability, Bayesian probability propagation and probabilistic information content}

Probabilistic cloud detection aims to evaluate the probability of cloud occurrence in a given observation $x$. Observations in this sense can be any observed property such as reflectance, brightness temperature, brightness temperature difference or reflectance ratio. For any observation an interval between values with a very high confidence for representing cloudfree background conditions $\left(x_{\mathrm{bg}}\right)$ and values with a very high confidence for representing cloud observations $\left(x_{\mathrm{cld}}\right)$ is defined. Figure 1 shows a schematic plot of the confidence interval concept. $x_{\mathrm{bg}}$ and $x_{\mathrm{cld}}$ can be either predefined or they are determined from the satellite observations, for example by averaging over larger observation windows. The cloud probability for each cloud test then follows the linear metric

$P_{\text {test }}=\frac{x-x_{\mathrm{bg}}}{x_{\mathrm{cld}}-x_{\mathrm{bg}}}$.

It can be assumed that any observation can be approximated by piecewise linearization around a traditional cloud mask threshold $x_{\text {binary }}$. Thus $x_{\text {cld }}$ and $x_{\text {bg }}$ must be selected to meet this assumption realistically, i.e., the interval $\left[x_{\mathrm{bg}}, x_{\mathrm{cld}}\right]$ must not be too large for the character of given observation $x$ and $x_{\text {cld }}=x_{\text {bg }}$ must also be avoided.

A linear model for assessing the cloud likelihood around $x_{\text {binary }}$ is chosen for the sake of convenience. The fact that it drops to zero at $x_{\mathrm{bg}}$ already suggests that there may be better suited probability models like the Gaussian cumulative probability distribution function. Nevertheless, for the purpose of cloud detection the linear model is a good tradeoff between accuracy and computational convenience (and thus efficiency).

Bayesian statistics are used to propagate cloud likelihood information within different tests to aggregate the information gained from the different inputs.

Generally Bayes' theorem states that the likelihood of one event can be calculated from the likelihood of all possible events and is traditionally used for updating probability information for very different purposes (e.g., Rodgers, 1998; MacKay, 2003).

The version of Bayes' theorem most often used in retrieval theory is

$P(x \mid y)=\frac{P(y \mid x) \cdot P(x)}{P(y)}$,

where $P(x)$ is the a priori probability of the information to be retrieved, $P(y)$ is the evidence of the information from the observation, $P(y \mid x)$ is the likelihood of observation $y$ given 


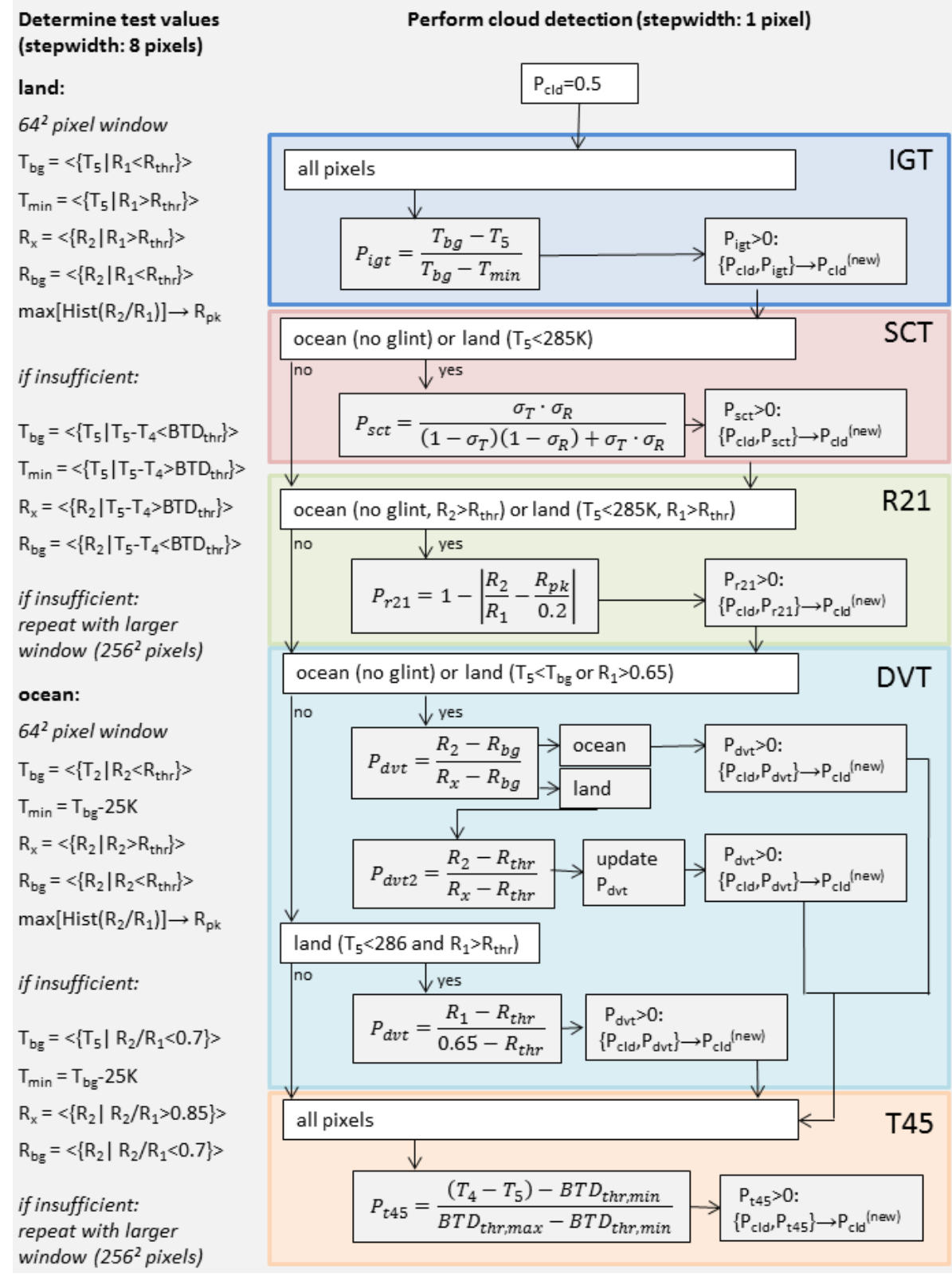

Figure 2. Flowchart of the APOLLO_NG cloud detection scheme.

the value of $x$ and $P(x \mid y)$ is the desired probability of the value for $x$ given the evidence $P(y)$.

In the framework of cloud detection, one can assume that the evidence carrying signal is a binary symmetric channel, which means that $P(\neg y)=1-P(y)$. This assumption becomes important when updating the probability. In this case, furthermore using $P(y)=P(y \mid x) P(x)+P(y \mid \neg x) P(\neg x)$, the updated probability of $x$ under the new evidence y becomes (MacKay, 2004)

$$
P(x \mid y)=\frac{P(x) \cdot P(y \mid x)}{(1-P(x)) \cdot(1-P(y \mid x))+P(x) \cdot P(y \mid x)},
$$

which is more convenient for the purpose of cloud detection than Eq. (2). The reason is that in Eq. (3) only two probability variables $(P(x)$ and $P(y \mid x))$ are evaluated and the first guess about the prior evidence $P(y)$ is carried in the probability evaluation as the sum of the conditional probabilities for $x$ being true and $x$ not being true.

As the information in all above described cloud tests is complementary, Eq. (3) is used for probability propagation if the respective likelihood $P(y \mid x)$ given by a cloud test is larger than zero (i.e., $P(y \mid x)>0$ ).

The thus determined $P_{\text {cld }}[=P(x \mid y)]$ describes the aggregated probability that an observation is cloud contaminated. 
The overall cloud probability stems from the test probabilities of very different cloud characteristics. So, although strictly speaking the five tests do not assess the same probability, their aggregation results in a good estimate of the probability that an observation really is cloud contaminated. In fact $P_{\text {cld }}$ represents the probability that an observation shows a cloud having all five characteristics (cold top, bright reflectance, reflectance ratio about one, spatially inhomogeneous and thin cloud, probably cirrus, at top). It is obtained by consecutive likelihood updates as described. Consequently, it can be used for cloud masking based on the desired confidence in either clear sky or cloudy pixel detection (see also discussion in Holzer-Popp et al., 2013).

In order to learn about the reliability of the cloud detection, the cloud probabilities (those of the five different cloud tests outlined above) can be used as input to an assessment of Shannon's information content $H_{\text {inf }}$ (Shannon and Weaver, 1949; Kolmogorov, 1968; Rodgers, 2000; MacKay, 2004). Therefore the probabilities for cloud observation have to be interpreted as independent signals. Five signals about the cloud state build the basis for the information content: $P_{\mathrm{IGT}}$, $P_{\mathrm{SCT}}, P_{\mathrm{DVT}}, P_{R_{21}}, P_{\mathrm{T} 45}$ (detailed mathematical descriptions follow in the sections below). In Shannon and Weaver (1949) the mathematical formulation of the information content requires that none of the probabilities equals 0 and also none equals one. Consequently, every $P_{x}=0$ would be set to 0.01 and every $P_{x}=1$ would be set to 0.99 for the purpose of calculating $H_{\text {inf }}$.

The probabilistic information content concept of Kolmogorov (1968) expands the view of Shannon and Weaver (1949) with respect to of Bayesian probability theory. Assuming that a priori information (i.e., information independent of the aforementioned cloud tests) is zero, the information content of the cloud detection algorithm emerges from the considerations of Kolmogorov (1968):

$H_{\mathrm{inf}}=-\sum_{j=1}^{5} P_{j} \cdot \log _{2}\left(P_{j}\right)$.

It is intuitive that the magnitude of $H_{\text {inf }}$ is related to the information carried by the vector of cloud test probabilities. High information content signifies that the different probabilistic cloud tests agree quite well. This is a direct consequence of the definition of information content. Assuming that the probabilities would highly disagree, including one of the tests with rather low probability would not add any new information to the knowledge about the cloud. In the sense of Shannon and Weaver (1949) all information about the cloud contamination would already be known by having the information of one or two tests. On the other hand, when the tests have similar probabilities, each provides additional information. Thus the resulting high information content indicates that all cloud tests contribute to the knowledge about the cloud presence. The confidence then increases that the pixel is quite contaminated. Consequently, high $H_{\text {inf }}$ re- lates to a more homogenous distribution of the probabilities. Low $H_{\text {inf }}$ indicates the significance of merely a single test for cloud detection. The more tests indicate cloudy (or cloudfree) conditions, the higher the confidence in the cloud detection. On the other hand, the original APOLLO was built in such a way that a single cloud test was able to classify an observation as cloudy. Consequently, $H_{\text {inf }}$ provides additional information about the cloud detection. Together with $P_{\text {cld }}$ it thus may be used to tune the output towards a more "clear confident" cloud mask or a more "cloud confident" cloud mask, depending on the purpose of the product.

\subsection{Probabilistic cloud detection tests}

\subsubsection{Infrared gross temperature (IGT)}

Thick or cold-topped clouds can easily be detected in infrared satellite imagery by their low brightness temperatures (e.g., Shenk and Curran, 1974; Saunders and Kriebel, 1988; Rosenfeld and Lensky, 1998; Frey et al., 2008). The infrared gross temperature test (IGT) makes use of the deviation between observed cloudy brightness temperature in one of the split window channels (centered at 11 and $12 \mu \mathrm{m}$ ) and the background temperature representing the surface. In order to evaluate the brightness temperature distance from the most likely cloud-free brightness temperature, in APOLLO_NG a $65 \times 65$ ( \pm 32 pixels) pixels box around a given observations is evaluated.

Over ocean surfaces, a reflectance ratio for the channels 1 and 2 (centered at 0.6 and $0.9 \mu \mathrm{m}$, respectively), which is lower than 0.7, indicates mainly cloud-free conditions (Kriebel et al., 2003). Frey et al. (2008) use channel 2 reflectance to identify cloud-free pixels with different confidence levels.

The channel 2 reflectance thresholds of Frey et al. (2008) with high confidence are used to filter all brightness temperatures in the $12 \mu \mathrm{m}$ channel $5\left(T_{5}\right)$ in the $65 \times 65$ pixel window and to determine the average brightness temperature of these most likely cloud-free data. The channel 2 reflectance thresholds are 0.03 over glint-free ocean and 0.26 over arid land surfaces. For vegetated land surfaces, a threshold of 0.14 for channel 1 reflectance is used (Frey et al., 2008) If insufficient dark pixels are found over ocean, then the corresponding reflectance ratio threshold from Kriebel et al. (2003) is used instead. If the $65 \times 65$ pixel window does not yield a valid background brightness temperature $T_{\mathrm{bg}}$, e.g., due to an insufficient number of pixels passing the filters, the window is expanded to a size of $257 \times 257$ pixels. Also the average brightness temperature threshold for cloud likelihood estimation $\left(T_{\min }\right)$ of the running window is estimated likewise. A channel 2 reflectance bound is set sufficiently high in order to approximate $T_{\min }$ by the maximum temperature of these supposedly cloudy pixels. That means in fact that each observation which has a channel 5 temperature colder than $T_{\min }$ is assumed to be cloud. 
The aforementioned approach can be understood as clustering the data into confident cloud free and supposedly cloudy data and subsequent cluster-averaging. In order to speed up the procedure, $T_{\mathrm{bg}}$ and $T_{\min }$ are only calculated for 1 in 8 pixels and linearly interpolated in between (assuming steady and slowly varying background and cloud field temperature conditions). IGT cloud probability $P_{\text {IGT }}$ is then calculated for each pixel as

$$
P_{\mathrm{IGT}}=\frac{T_{\mathrm{bg}}-T_{5}}{T_{\mathrm{bg}}-T_{\mathrm{min}}} .
$$

If $T_{5} \leq 233.15 \mathrm{~K}$ and $T_{\mathrm{bg}} \leq 233.15 \mathrm{~K}$ (homogeneous freezing threshold, Pavolonis and Heidinger, 2004) it can be assumed that the target is a synoptic scale convective system, when also $R_{1} \geq 0.4$. Then $P_{\text {IGT }}$ is set to 0.95 without evaluating Eq. (5) as in large scale synoptic systems it may be extremely difficult to find the appropriate background temperature $T_{\mathrm{bg}}$. The value of 0.95 is an arbitrary choice in order to allow subsequent tests to include cloud information, which would not be possible as a consequence of Eq. (3) if $P_{\text {IGT }}$ would be set to 1 or even 0.99 .

\subsubsection{Spatial coherence test (SCT)}

A spatial coherence test is used to examine regions with high variability in either reflectance or brightness temperature (Saunders and Kriebel, 1988; Kriebel et al., 2003). In the APOLLO_NG algorithm the spatial coherence is evaluated consecutively in temperature and reflectance data during day. It starts with evaluating the $3 \times 3$ pixel standard deviation of the brightness temperature field $\left(\sigma_{T_{5}}\right)$ and of the channel 2 reflectance field $\left(\sigma_{R_{2}}\right)$. Coastal pixels are always inhomogeneous by definition and are thus discarded in the SCT likelihood. Moreover, over land the SCT is only applied when $P_{\mathrm{IGT}}>0$.

Applying Eq. (3) with likelihoods $L_{T_{5}}=\sigma_{T_{5}} / \mathrm{NP}_{T_{5}}$ and $L_{R_{2}}=\sigma_{R_{2}} / \mathrm{NP}_{R_{2}}$ the respective $\mathrm{SCT}$ cloud probability $P_{\mathrm{SCT}}$ is then evaluated as

$$
P_{\mathrm{SCT}}=\frac{L_{T_{5}} \cdot L_{R_{2}}}{\left(\left[1-L_{T_{5}}\right] \cdot\left[1-L_{R_{2}}\right]+L_{T_{5}} \cdot L_{R_{2}}\right)} .
$$

Cloud likelihood normalization parameters of $\mathrm{NP}_{T_{5}}=$ $1.0 \mathrm{~K}$ and $\mathrm{NP}_{R_{2}}=0.2$ are used for the standard deviations of $T_{5}$ and $R_{2}$ (values adapted from Kriebel et al., 2003). Physically that means, the higher the standard deviation (the more variable the reflectance respective temperature field), the higher is the likelihood that the window is cloud affected and is mostly sensitive to broken or inhomogeneous cloud fields within the observation window. One should be aware that the standard deviation of the reflectance field is also subject to effects of variability of the bidirectional reflectance distribution function (BRDF) of the surface over the $3 \times 3$ pixel box. It is assumed that in the presence of clouds these effects are minor compared to the impact of bright and inhomogeneous clouds given the small size of the sample.

\subsubsection{Dynamic visible test (DVT)}

Clouds can be identified as bright reflecting objects in satellite images (e.g., Shenk and Curran, 1974). Many different approaches have been identified to use reflectance thresholds for cloud discrimination (examples listed in e.g., Nakajima and Kaufman, 1993, Frey et al., 2008, and Klüser et al., 2008). The dynamic visible test uses dynamic thresholds based on channel 2 (over ocean) or channel 1 (over land) reflectance histograms for cloud identification. The approach is analogous to the IGT approach, but the scaling is performed with minimum cloudy and maximum clear sky reflectance for given confidence levels (Frey et al., 2008 as for IGT, see Sect. 2.3.1). Consequently, the DVT cloud likelihood is formulated as

$P_{\mathrm{DVT}}=\frac{R_{1,2}-R_{\mathrm{bg}}}{R_{\mathrm{bg}}-R_{\max }}$

and $R_{\mathrm{bg}}$ and $R_{\max }$ are again evaluated for land and ocean separately. Applying only Eq. (7) with the cluster-based background and maximum reflectance values would result in high cloud probabilities over various land surfaces such as deserts or other bright surfaces. Thus the cloud probability is updated with Eq. (3) by using a priori background values for arid and non-arid land surfaces depending on the observed scenery. Following Frey et al. (2008) land surfaces are distinguished between arid and non-arid (i.e., vegetated) by the minimum channel 1 reflectance in the running window. If the minimum reflectance falls below the clear sky value (high confidence) for non-arid land or the brightness temperature of the background is lower than $285 \mathrm{~K}$, the filtering uses the non-arid values and the arid boundary thresholds are used otherwise. Any residual misinterpretation of desert surface properties for cloud probability is excluded by flagging all pixels that have non-negative $P_{\mathrm{DVT}}$, are warmer than $278 \mathrm{~K}$, darker than 0.6 and have negative split window brightness temperature difference of $T_{4}-T_{5}$ (see e.g., Klüser and Schepanski, 2009). Also very warm pixels $\left(T_{2}>290 \mathrm{~K}\right)$ for which the reflectance ratio test (see Sect. 2.3.4) yields zero probability are discarded over land (a discussion on warm top clouds can also be found in Holzer-Popp et al., 2008). For these desert pixels $P_{\mathrm{DVT}}$ is set back to 0 in the case that the reflectance is lower than 0.65 .

Over water bodies the DVT test is also sensitive to sunglint. Thus the DVT is not applied within the area potentially affected by sunglint, which can be determined from theoretical considerations (Saunders and Kriebel, 1988).

\subsubsection{Shortwave reflectance ratio $\left(R_{21}\right)$}

Due to the typical optical properties of clouds the ratio of shortwave reflectance (i.e., between the AVHRR channels 2 and 1 centered around 0.9 and $0.6 \mu \mathrm{m}$, respectively) provides information about the likelihood of clouds compared to open water bodies or land surfaces. Clouds usually occupy 
reflectance ratios between 0.7 and 1.3 (Kriebel et al., 2003). The peak of the reflectance ratio histogram in the moving window is determined according to the description by Saunders and Kriebel (1988). The distance of the actual observation from the peak is used to calculate $P_{R_{21}}$, if it is smaller than 0.2 (that widens the original threshold of Saunders and Kriebel, 1988, in order to allow for a wider range of probabilities):

$P_{R_{21}}=\frac{\left|\left(R_{2} / R_{1}\right)-\left(R_{2} / R_{1}\right)_{\text {peak }}\right|}{0.2}$.

The reflectance ratio is used for cloud probability also over land (requiring that $T_{5}<285$ and $T_{4}-T_{5}>0$ in order to exclude warm desert surfaces). The $R_{21}$ test is not executed in the sunglint area.

\subsubsection{Brightness temperature difference $\left(T_{45}\right)$}

The test for thin clouds and cirrus is one of the most important for a couple of applications including aerosol retrieval (e.g., Holzer-Popp et al., 2013). Thin clouds can be detected by their differential influence on brightness temperatures at 11 and $12 \mu \mathrm{m}$ (the so called split window channels) due to slopes in the complex refractive index of water and ice in that spectral region (e.g., Warren, 1984). Especially for cirrus clouds the extinction efficiency is much lower at 11 than at $12 \mu \mathrm{m}$ (Yang et al., 2005). The brightness temperature difference (BTD) of AVHRR channels 4 and 5 has been used frequently for cirrus detection and visualization (e.g., Rosenfeld and Lenksy, 1998; Frey et al., 2008).

The thresholds for thin cloud detection vary with observed channel 4 brightness temperature as well as with the cosine of the viewing zenith angle $\Theta_{\mathrm{v}}$ (Saunders and Kriebel, 1988). In order to determine the thin cloud probability from the $T_{45}$ test the BTD thresholds of Saunders and Kriebel (1988) are evaluated for a range of conditions enveloping the actual conditions (namely, $T_{4}$ and $\cos \left(\Theta_{\mathrm{v}}\right)$ ). From these envelope conditions the minimum and maximum BTD (named BTD $_{\text {min }}$ and $\mathrm{BTD}_{\max }$, respectively) are determined and the thin cloud probability is evaluated as for the other tests:

$P_{T_{45}}=\frac{\mathrm{BTD}-\mathrm{BTD}_{\min }}{\mathrm{BTD}_{\max }-\mathrm{BTD}_{\min }}$.

\subsubsection{Additional nighttime probability estimates $\left(T_{43}\right.$ and $T_{35}$ )}

At night, the channels 1 and 2 cannot be exploited for cloud detection, as no reflected sunlight is available. Nevertheless, the channel 3 of AVHRR is available at night for all three AVHRR versions. For the AVHRR/1 and AVHRR/2 instruments, the channel 3 is permanently centered at $3.7 \mu \mathrm{m}$, while for AVHRR/3 the channel 3 mode is switched between $1.6 \mu \mathrm{m}$ at day and $3.7 \mu \mathrm{m}$ at night. Instruments such as SEVIRI, AATSR or VIIRS operate both, 1.6 and 3.7 (or 3.9) $\mu \mathrm{m}$ channels permanently. Consequently, for these instruments the 3.7 (3.9) $\mu \mathrm{m}$ brightness temperature is used as additional input at night. Following Kriebel et al. (2003) the brightness temperature differences between channel 4 and channel 3 and between channel 3 and channel 5 are exploited during night ( $T_{43}$ respective $T_{35}$ ). The lower and upper boundaries $\left(\mathrm{BTD}_{\min }\right.$ respective $\mathrm{BTD}_{\max }$ in Eq. 9) of the probability estimation interval are 0.5 and $1.5 \mathrm{~K}$ in the first and 3 and $5 \mathrm{~K}$ in the latter $(1 \mathrm{~K}$ respective $2 \mathrm{~K}$ confidence interval around the thresholds used in Kriebel et al., 2003).

\section{Snow discrimination}

The a posteriori snow detection (i.e., detection of falsely identified clouds in the case of snow cover) follows the same probabilistic approach as the cloud detection scheme. That is, the binary thresholds in Kriebel et al. (2003) and Gesell (1989) are expanded by the methodology explained above in order to yield a snow contamination probability. The snow discrimination is only applied over land and for observations with $258 \mathrm{~K} \leq T_{5} \leq 278 \mathrm{~K}$. Also $\Theta_{s}<85^{\circ}, P_{T_{45}}=$ 0 and $P_{\mathrm{DVT}}>0$ are necessary prerequisites for successful snow detection. That means, the pixel must be illuminated by sun, must not be cirrus contaminated and the dynamic visible reflectance test must indicate cloud. Then the snow detection is performed applying Eq. (7) to the channel 3 reflectance $R_{3}$. The boundary thresholds are $R_{\mathrm{bg}}=0.1$ and $R_{\max }=0.03$ (which only becomes positive when $R_{3}<R_{\mathrm{bg}}$ ) in the case of channel 3 being centered at $3.7 \mu \mathrm{m}$. Otherwise, i.e., channel 3 center wavelength of $1.6 \mu \mathrm{m}, R_{\mathrm{bg}}=0.15$ and $R_{\max }=0.06$. The snow probability is updated once again by using the reflectance ratio between channels 1 and 3 with the confidence interval boundaries $R_{\mathrm{bg}}=20, R_{\max }=15$ for the $3.7 \mu \mathrm{m}$ channel and $R_{\mathrm{bg}}=6.67, R_{\max }=5$ for the $1.6 \mu \mathrm{m}$ channel.

\section{Cloud property retrieval}

\subsection{Optical depth and effective radius}

The mathematics of cloud property retrieval in the original APOLLO follow the approach outlined in Stephens (1978). The general approach and the mathematical treatment have widely been conserved, but a couple of improvements and innovations have gone into the realization of the cloud properties retrieval for APOLLO_NG. Moreover also for cloud properties a probabilistic treatment has been implemented in APOLLO_NG. While in Stephens (1978) absorption was used only for angular correction of the red channel reflectance, now the contrast between one non-absorbing and one absorbing channel ("absorbing" here means that cloud droplets absorb at the respective wavelengths) is used for inferring optical depth and effective radius (Stephens, 1984; Nakajima and King, 1990). 

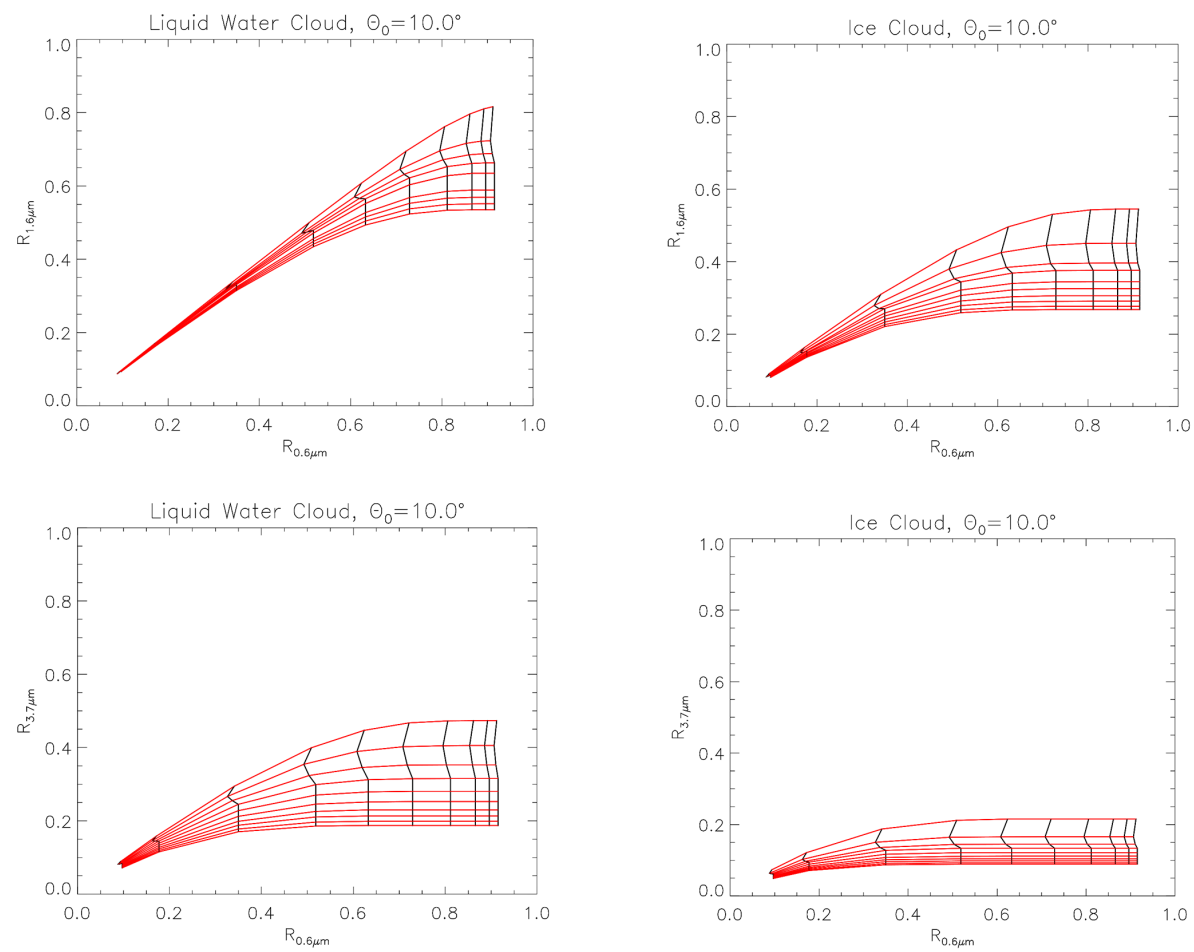

Figure 3. Reflectance of liquid water (left) and ice (right) clouds at the absorbing versus the non-absorbing channel for the absorbing channel being centered at $1.6 \mu \mathrm{m}$ (top) and $3.7 \mu \mathrm{m}$ (bottom). Cloud reflectance is simulated with the two-stream scheme of Coakley and Chylek (1975) for a sun zenith angle or $10^{\circ}$ and for various optical depths and effective radii (see text for details).

As inherited from the original APOLLO it is assumed that the solution of the radiative transfer problem used in traditional schemes does a reasonably good job for AVHRR. In fact the signal-to-noise ratio as well as the broad spectral response functions of the solar bands of AVHRR (together with the calibration) suggest that using a two-stream approximation will provide reasonably good results. In APOLLO_NG the two-stream solution of the radiative transfer problem as described in Coakley and Chylek (1975) is used for the nonabsorbing and the absorbing channel. This scheme is identical to the scheme used for the original APOLLO cloud property retrieval (Kriebel et al., 1989) and thus truly complies with the APOLLO legacy. The surface is assumed to be Lambertian with the albedo estimated from the cloud-free surroundings as described in Sect. 2.3.3.

The two-stream approach used in APOLLO_NG offers the potential to calculate reflectance online at reasonably high performance. This gives the advantage that the method becomes independent of sensor-specific radiance lookup tables and tuning factors (as long as the channel specific cloud single scattering albedo and asymmetry parameter are provided, both essential inputs to the two-stream approximation) - of course at the price of reduced accuracy (see discussion in Coakley and Chylek, 1975). The second advantage is that the calculations can be performed online for a range of optical depths and effective radii, so that the proba- bilistic potential can be fully exploited. It nevertheless has to be made absolutely clear that the two-stream approach buys the mathematical simplicity at the price of losing accuracy in the results. That becomes of larger importance for sensors with better calibrated solar channels having narrow filter functions. Consequently, the results obtained by using the two-stream approach as outlined below should be seen as rough estimates in the case of instruments such as SEVIRI, AATSR or VIIRS. They are used here for the sake of consistency with the AVHRR methodology and not because we think they will offer high precision. Moreover, as the traditional APOLLO scheme has been shown to be useful for example in solar irradiance calculations (Oumbe et al., 2013) it is envisaged to keep the physics of the traditional APOLLO scheme. The new implementation in APOLLO_NG compared to APOLLO thus mainly consists of incorporating the effective radius retrieval and the probabilistic approach also yielding uncertainties for the retrieved parameters.

Figure 3 exemplarily shows the simulated reflectance in the absorbing and non-absorbing channels of AVHRR for a solar zenith angle of $10^{\circ}$ and different pairs of effective radius and optical depth. The red curves connect calculation results with constant effective radius. The black curves indicate constant optical depth, which is mostly related to the reflectance of the non-absorbing channel (Stephens et al., 1984; Nakajima and King, 1990). Orthogonality in the red 
and black curves would correspond to the possibility of retrieving optical depth and effective radius independently, as is more or less the case for very high optical depths. At lower optical depth the sensitivity to effective radius changes decreases, and as a result the uncertainty in retrieved effective radius will become much higher.

For the $3.7 \mu \mathrm{m}$ channel of AVHRR under daylight conditions it is essential to correct for the thermal emission in order to calculate the reflected part of the signal. Figure 3 shows that getting an improper reflectance in the absorbing channel will cause large errors in the retrieved effective radius, but also in the optical depth. The method of Kaufman and Nakajima (1993) also takes into account above-cloud water vapor absorption for calculation of $3.7 \mu \mathrm{m}$ reflectance and is applied within APOLLO_NG.

The simultaneous retrieval of cloud optical depth and effective radius (Nakajima and King, 1990) is performed consecutively for each observation for potential liquid water and ice phase clouds. The approach is to calculate optical depths with the equations of Coakley and Chylek (1975) and Stephens et al. (1984) from a set of 10 different reflectance values $R_{1, \text { sim }}$ in the red (non-absorbing) channel ranging from 0.05 to 0.95 . For each of these 10 values for $R_{1 \text {,sim }}$ the distance to the observation $R_{1}$ is determined. The weighting factor for each $R_{1, \text { sim }}$ is then calculated assuming a Gaussian distribution around the observation $R_{1}$ with a standard deviation of $10 \%$. A first guess optical depth is determined by weighting the optical depth values associated with each reflectance value by the thus determined Gaussian weighting factor. Having obtained first guess optical depth, the same procedure is repeated with a set of 10 different effective radii for the reflectance in the absorbing channel, yielding $R_{3, \text { sim }}$. The Gaussian distribution of the thus simulated reflectance around the observation $R_{3}$ provides the weighting factors for the effective radius. The resulting effective radius (evaluated for assumed liquid water and ice clouds separately) is again calculated by weighting the input values with the weighting factors. Moreover the first guess optical depth is once again corrected for the influence of the effective radius on the nonabsorbing reflectance (through the phase function and thus the backscattered fraction).

Starting values for the effective radius are $2 \mu \mathrm{m}$ through $20 \mu \mathrm{m}$ in $2 \mu \mathrm{m}$ steps for liquid water clouds and $10 \mu \mathrm{m}$ through $55 \mu \mathrm{m}$ in $5 \mu \mathrm{m}$ steps for ice clouds. Necessary single scattering albedo and asymmetry parameter (see Coakley and Cox, 1975; Stephens et al., 1984) are determined with Mie calculations for liquid water clouds. For the sake of convenience and as the results for different effective radius values are combined in the probabilistic scheme, we performed the Mie simulations for mono-disperse liquid-water clouds. For ice clouds the optical properties of Baum et al. (2014) are used.

Within the APOLLO_NG scheme not only the mean values for optical depth and effective radius are calculated, but also the standard deviation of the thus-obtained probability distributions. This methodology easily allows for estimating the uncertainty of the retrieved quantity. Consequently, each retrieval is accompanied by an uncertainty estimate, which is a prerequisite e.g., for data assimilation in numerical models.

\subsection{Cloud top phase, cloud top temperature and cloud water path}

During the previous step two values for cloud optical depth and effective radius have been obtained: one for purely liquid phase clouds and one for ice clouds. Cloud phase discrimination yields the final value for optical depth and effective radius through representative weighting following the probabilistic approach. Therefore the Gaussian distributions for the weighting factors of the simulated channel 3 reflectances (depending on the selected effective radii) are used for evaluating the likelihood of liquid water respective ice clouds. As the absorbing channel is sensitive to the cloud phase as well as to the effective radius, the $R_{3 \text {,sim }}$ forward simulations are also well suited for cloud phase assessment. It is assumed that the sum over all distance weighting factors is smaller for the cloud phase better representing the observed cloud. Consequently, the first guess of the liquid phase fraction is simply calculated as

$\mathrm{LPF}_{1}=\frac{\sum_{j} \phi_{j} \text { (liquid) }}{\sum_{j} \phi_{j} \text { (liquid) }+\sum_{j} \phi_{j} \text { (ice) }}$.

$\phi_{j}$ denotes the weighting factor for the $j$ th effective radius value for liquid water or ice clouds respectively. If no cirrus cloud is detected, i.e., if the topmost cloud layer is assumed to be opaque, the liquid phase fraction is once more updated with the likelihood of glaciated cloud droplet expressed as a function of temperature. Therefore it is assumed that below the homogeneous freezing temperature of $233.15 \mathrm{~K}$ all droplets at cloud top are frozen and that above the pure water freezing point of $273.15 \mathrm{~K}$ all cloud droplets are liquid (Pavolonis and Heidinger, 2004). Thus applying Eq. (3) with these boundary thresholds yields an updated liquid phase fraction (LPF) of

$$
\begin{aligned}
& \mathrm{LPF} \\
& =\frac{\mathrm{LPF}_{1} \cdot\left(\frac{T_{5}-233.16 \mathrm{~K}}{40 \mathrm{~K}}\right)}{\left(1-\mathrm{LPF}_{1}\right) \cdot\left(1-\frac{T_{5}-233.16 \mathrm{~K}}{40 \mathrm{~K}}\right)+\mathrm{LPF}_{1} \cdot\left(\frac{T_{5}-233.16 \mathrm{~K}}{40 \mathrm{~K}}\right)}
\end{aligned}
$$

for any observation with $233.15 \mathrm{~K} \leq T_{5} \leq 273.15 \mathrm{~K}$. Moreover for $T_{5}>273.15 \mathrm{~K}, \mathrm{LPF}=1$ and for $T_{5}<233.15 \mathrm{~K}$, $\mathrm{LPF}=0$. Cloud optical depth and effective radius are then finally determined by weighting the results for liquid clouds by LPF and those for ice clouds by (1- LPF).

Once cloud optical depth, effective radius and liquid phase fraction are determined, cloud top temperature and cloud water path can be calculated. Cloud top temperature is estimated from $T_{5}$ and cloud optical depth by inverting the relationship 
between cloudy and clear radiance $\left(I_{\mathrm{cld}}\right.$ and $I_{\mathrm{clr}}$, respectively) and the channel 5 radiance $I_{5}$. The corresponding equation (e.g., Guignard et al., 2012)

$1-\exp (-\tau)=\frac{I_{2}-I_{\mathrm{clr}}}{I_{\mathrm{cld}}-I_{\mathrm{clr}}}$

can easily be solved for $I_{\text {cld }}$. It directly yields cloud top temperature through inversion of the temperature-radiance relationship. $I_{\mathrm{clr}}$ is approximated by the background temperature value $T_{\mathrm{bg}}$ as determined for the IGT cloud probability. Infrared optical depth $\tau_{\text {ir }}$ is different from visible optical depth $\tau_{\text {vis }}$ for non-opaque clouds (e.g., Comstock et al., 2007; Baum et al., 2014). Here the approximation of Chang and $\mathrm{Li} \mathrm{(2005)} \mathrm{is} \mathrm{used,} \mathrm{which} \mathrm{relates} \tau_{\text {ir }}$ to $\tau_{\text {vis }}$ by

$\tau_{\text {ir }}=\frac{\tau_{\text {vis }}}{\mathrm{LPF} \cdot 2.13+(1-\mathrm{LPF}) \cdot 2.56}$.

Cloud water path can be expressed as a function of optical depth and effective radius as well as extinction efficiency $Q_{\mathrm{e}}$. For spherical liquid phase cloud droplets $Q_{\mathrm{e}} \rightarrow 2$, while for ice clouds the extinction efficiency is determined by effective radius (and the crystal shape) and thus is determined from the weighting factors of the effective radius from the optical properties database. Liquid phase and ice phase extinction efficiencies are weighted according to the liquid phase fraction (the same is done for density $\rho$ ). Then the cloud water path is calculated as

$\mathrm{CWP}=\frac{4 \cdot \rho \cdot \tau_{\mathrm{vis}} \cdot r_{\mathrm{eff}}}{3 \cdot Q_{\mathrm{e}}}$.

Besides the liquid phase fraction (or ice phase fraction $\mathrm{IPF}=1-\mathrm{LPF}$ ) also a cloud top phase identifier is derived in order to facilitate the interpretation and application of APOLLO_NG results. It is widely controlled by LPF and CTT as well as $P_{T_{45}}$.

As a starting point all observations having CTT $>273.15 \mathrm{~K}$ or LPF $>0.75$ are identified as liquid water clouds. Correspondingly, all clouds having CTT $\leq 273.15 \mathrm{~K}$ and $\mathrm{LPF}<0.05$ are initially set to opaque ice clouds. If CTT $\leq$ $273.15 \mathrm{~K}$ and $0.05 \leq \mathrm{LPF} \leq 0.75$ the clouds are classified as supercooled liquid/mixed phase. In the case that $P_{T_{45}}$ indicates thin cloud, presence of cirrus is assumed if $T_{5}>233.15$ (i.e., the cloud is not opaque with cold-top such as deep convective clouds) and CTT $<253.15 \mathrm{~K}$ (i.e., the cloud top is reasonably cold for cirrus). Given cirrus has been identified but the optical depth is larger than 2 and more than one test indicates the presence of clouds (i.e., $H_{\mathrm{inf}}>0$ ) it is assumed that more than one cloud layer is present and the overlap flag is set.

In the cloud detection scheme the cloud fraction of each pixel is approximated by the cloud probability as a starting point. Thus observations with $P_{\text {cld }}<95 \%$ are assumed to be partially cloudy. That follows the legacy of the original APOLLO scheme. During the cloud property retrieval the fractional cloud cover is estimated from the average of the first guess cloud fraction of a surrounding $3 \times 3$ pixel box. Hence it is assumed that near cloud edges the information carried by neighboring pixels translates into subpixel cloudiness (see e.g., Koren et al., 2008; Hirsch et al., 2014).

\section{Example results from AVHRR}

Figure 4 depicts the RGB composite and cloud mask in the traditional APOLLO quicklook style for an overpass of NOAA-18 over Europe in HRPT (High Resolution Picture Transmission) projection with $1.1 \mathrm{~km}$ spatial resolution at nadir received at the receiving station of the German Aerospace Center (DLR) at Oberpfaffenhofen, South Germany, for 15 July 2008. In the cloud mask image fully cloudy pixels are white, partially cloudy pixels grey, sunglint is yellow, cloud-free water is blue and cloud-free land brown (see Kriebel et al., 2003). Both images have been produced with APOLLO_NG strictly following the tradition of APOLLO. The cloud mask derived from APOLLO_NG and depicted in Fig. 4 includes all pixels with cloud probability larger than $25 \%$.

Figure 5 shows an example of APOLLO_NG cloud probability $P_{\text {cld }}$ and the corresponding cloud detection information content $H_{\text {inf }}$ for the AVHRR scene on 15 July 2008 in isotropic Mercator projection with $1 / 30^{\circ}$ grid resolution. Clouds have been detected over wide parts of central and northern Europe with high confidence. The information content on the other hand shows that not in all cases with high cloud probability all cloud tests agree as the variability in $H_{\text {inf }}$ is much higher than the variability in $P_{\text {cld }}$. Given the dependences of the different cloud tests, it is not surprising that the information content is highest for cumulus and stratocumulus cloud fields with high spatial variability (e.g., northwest of Great Britain and over central Europe) and not that high for the cyclonic deep convection in the vicinity of Iceland. In the latter case ice-topped cold clouds with rather high spatial homogeneity prevail, reducing the suitability of the SCT, $R_{21}$ and $T_{45}$ tests. Comparing Fig. 4 (the cloud mask derived from $P_{\text {cld }}>25 \%$ ) and Fig. 5 shows that there are regions where cloud probability is low (for example $<25 \%$ ) and it is not obvious from visual inspection of the RGB image that these are indeed cloud contaminated. Such regions are for example found in central France, over North Africa and over the Mediterranean Sea. Consequently, an adjustment of the clear sky threshold for $P_{\text {cld }}$ to $25 \%$ results in a larger number of clear sky pixels compared to what would be flagged with a threshold of $P_{\text {cld }}>0 \%$. Such a possibility of adjustment is especially important for the purpose of aerosol retrieval (Holzer-Popp et al., 2013) and solar irradiance estimation.

For the retrieval of cloud products a minimum cloud probability of $25 \%$ has been used without any constraint on $H_{\text {inf. }}$. That means the cloud mask from Fig. 4 has been used. Thus 

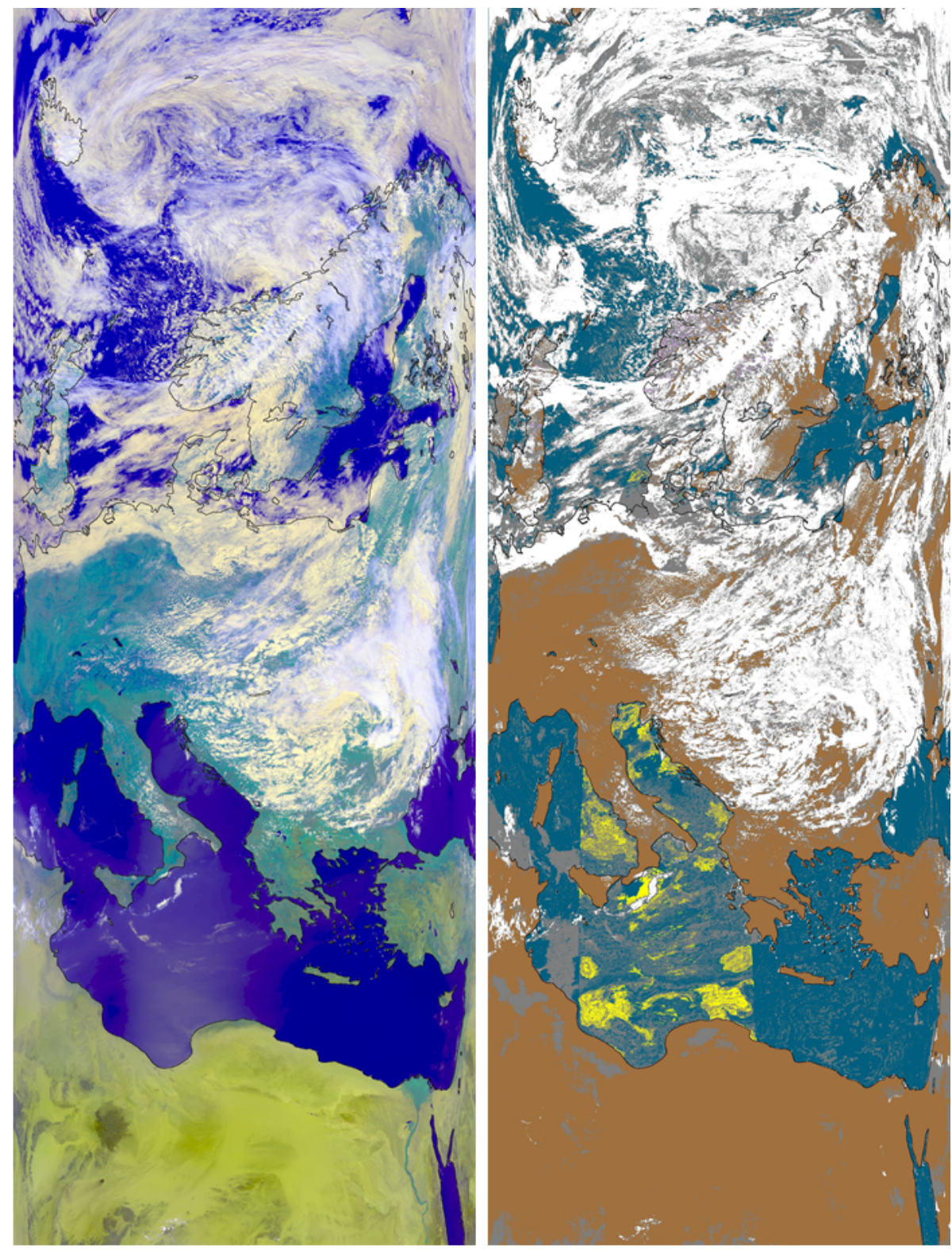

Figure 4. RGB composite and cloud mask in the traditional APOLLO quicklook style (Kriebel et al., 2003; see also text for color description) from AVHRR on NOAA-18 for 15 July 2008 in orbit projection.

the resulting cloud mask in this example is a bit more on the clear conservative side than on the cloud conservative side. Recent analysis of SEVIRI cloud detection suggests that the cloud mask of the traditional APOLLO scheme is best represented by setting a cloud probability of $40 \%$ (not shown). That value may change with sensor, as the traditional APOLLO versions slightly vary with sensor. It is another indicator that the $25 \%$ minimum $P_{\text {cld }}$ threshold used for generating the cloud mask in Fig. 4 and subsequently for deriving the cloud products (Fig. 6) is rather clear conservative.

Figure 6 shows the resulting cloud optical depth and effective radius for the same AVHRR scene together with their associated uncertainties. It is evident that the relative uncertainty for cloud optical depth increases with optical depth, especially in cold top (ice) clouds, as can easily be understood from the reduced sensitivity of $R_{1}$ to optical depth for high $\tau_{\text {vis }}$ (Fig. 3) in the case of ice cloud top phase. Moreover high relative uncertainties arise from very low optical depths. Pure ice phase clouds can easily be detected in Fig. 6 by large effective radii (i.e., $R_{\text {eff }}>40 \mu \mathrm{m}$ ). While the retrieved effective radius is very sensitive to the ice and liquid water fractions derived during the retrieval, Fig. 6 also nicely shows this sensitivity by the presence of effective radii between 20 and $30 \mu \mathrm{m}$. Also for cloud top effective radius, the highest 

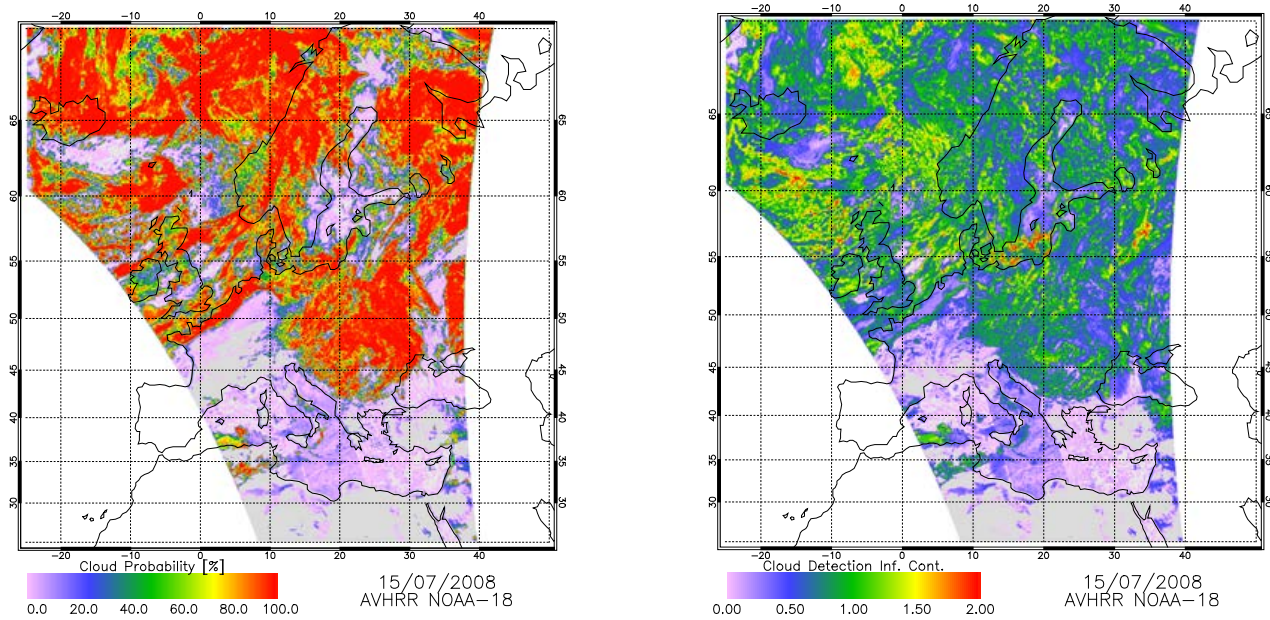

Figure 5. Cloud probability (left) and cloud detection information content (right) from AVHRR on NOAA-18 for 15 July 2008.
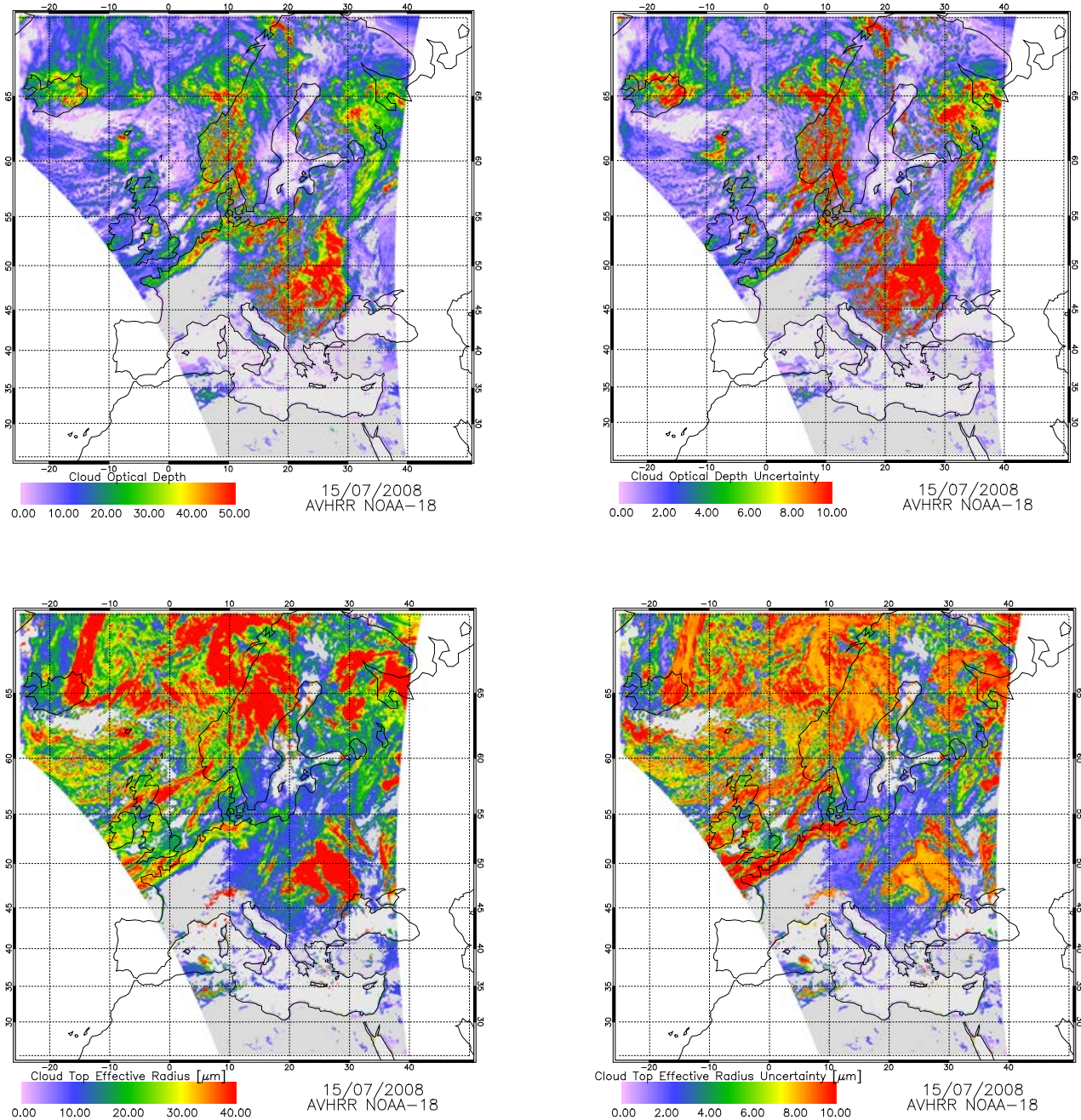

Figure 6. Cloud optical depth (top row) and effective radius (bottom row) retrieval results (left) and associated uncertainties (right) for the same data as in Fig. 4. 

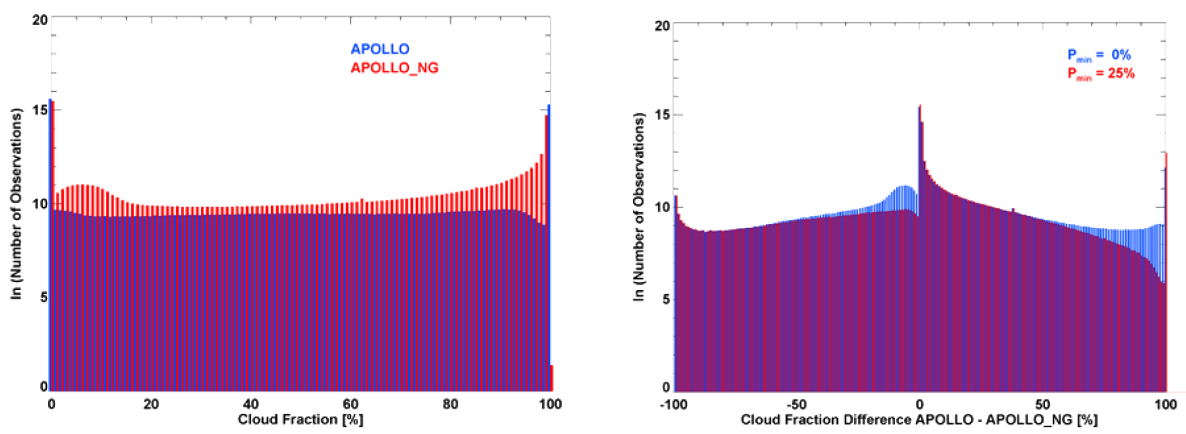

Figure 7. Left - cloud fraction histograms of APOLLO (red) and APOLLO_NG (blue) for the central overpass of NOAA-19 on 15 July 2013. Right - histograms of the cloud fraction difference between APOLLO and APOLLO_NG for all cloud probability values (blue) and with all observations having cloud probability smaller than $25 \%$ set to cloud free (red).

uncertainties are observed for high effective radii, i.e., for ice clouds, or in cases of low cloud optical depth.

In Figure 7 histograms of cloud fraction for one AVHRR overpass over Europe (15 July 2013, daytime) are presented for APOLLO_NG as well as for the original APOLLO scheme. It can be seen that APOLLO tends to detect much more either fully cloudy or entirely cloud-free pixels (the population of the histogram bins are depicted as the natural logarithm of the numbers of observations), whereas APOLLO_NG has higher shares of cloud fractions between 1 and $99 \%$. The histograms of the cloud fraction differences between APOLLO and APOLLO_NG are as well presented. Here the blue bars indicate the difference distribution when all pixels are taken into account regardless of the APOLLO_NG cloud probability, and also when APOLLO_NG pixels with cloud probabilities smaller than $25 \%$ are set to cloud free (red bars). It is evident that in the latter case the amount of rather large positive differences (i.e., APOLLO has much higher cloud fraction than APOLLO_NG) decreases. The two histograms exemplarily show the potential benefit of changing the minimum cloud probability for cloud detection. Although in Fig. 7 only one springtime overpass over Europe covering a wide range of complex cloud conditions is analyzed, histograms from other overpasses in all four seasons show very similar results. This comparison should not be seen as a rigorous validation, but it gives a first indication about the performance of the APOLLO_NG cloud detection. Referring to the evaluation of the traditional APOLLO scheme, $70 \%$ of cloud cover retrievals showed agreement within $\pm 1 / 8$ cloud amount when compared to SYNOP observations (Kriebel et al., 2003). The comparison of APOLLO and APOLLO_NG shows agreement of $78.4 \%$ (all observations) to $79.3 \%$ (minimum cloud probability of $25 \%$ ) within $\pm 1 / 8$ (cloud fraction $\pm 12.5 \%$ ).

\section{Discussion}

The examples presented above showcase the possibility of using the original APOLLO cloud detection metrics and physical parameterizations in a probabilistic manner. Such an approach increases the information gained from AVHRR observations. With the probabilistic approach the desired degree of conservativeness in the cloud detection can be tuned from clear confident to cloud confident. This is achieved by selecting an appropriate minimum cloud probability for identifying cloud contaminated pixels. In applications of cloud property remote sensing the minimum probability will be set rather high. Contrastingly in cloud masking applications for clear sky remote sensing purposes, such as for example aerosol retrieval, the minimum probability will be selected rather low. Each evaluation of the cloud mask must thus take into account the corresponding purpose as well as the selection of minimum probability. In Fig. 4 it has been shown that cloud probability alone will result in a cloud mask which can be adapted to the purpose of usage (see Fig. 7), but the quality of the cloud detection can furthermore been assessed by the interpretation of the Shannon information content $H_{\text {inf }}$ provided along with $P_{\text {cld }}$. The more cloud tests detect potential cloud contamination and thus the higher $H_{\text {inf }}$, the more confidence one can have in the retrieved cloud probability (even if it is a low probability).

Using the Stephens et al. (1984) extension of the original cloud property parameterization scheme allows simultaneous retrieval of cloud effective radius and optical depth from a two-channel approach like the often-used method of Nakajima and King (1990). This is a major update compared to the original APOLLO scheme, where cloud effective radius was assumed to be constant at $10 \mu \mathrm{m}$ (e.g., Klüser and Holzer-Popp, 2010). Also the physical cloud products are determined in a probabilistic approach using the distances of online simulations to the observations as a first guess for the probability distribution of cloud properties. In order to be able to perform the simulations online, the two-stream approximation already implemented in the original APOLLO 
is also used in APOLLO_NG, extended by the formulation of the two-stream equations for absorbing channels (Coakley and Chylek, 1975). We are fully aware that using a twostream approximation will reduce the precision of the reflectance simulations. Nevertheless, performing the simulations online is seen to be advantageous over the loss of precision for the purpose of APOLLO_NG. Moreover, simulating the $3.7 \mu \mathrm{m}$ reflectance would require very good knowledge about the cloud top temperature a priori. This knowledge typically is not available. Consequently, the errors brought about by the estimation of the thermally emitted part of the $3.7 \mu \mathrm{m}$ band radiance would again result in large errors which would remove all advantages from the precise radiative transfer modeling. This would not be a large problem for sensors such as SEVIRI or VIIRS which also have absorbing channels at 1.6 or $2.2 \mu \mathrm{m}$. For the AVHRR/1 and AVHRR/2 with the $3.7 \mu \mathrm{m}$ channel the errors introduced by the use of the two-stream approximation are expected to not be larger than those of the other estimations and assumptions. Especially the conversion from thermal-corrected radiance to reflectance includes the assumption of Lambertian reflection and potentially introduces additional errors. Also, given the rather broad channel response functions of the AVHRR instruments, the uncertainties of the two-stream approximation might be acceptable compared to their influence for instruments with finer resolution. In contrast to the traditional APOLLO scheme the APOLLO_NG implementation rigorously follows a modular programming approach. Consequently, the two-stream scheme could easily be replaced with a more precise forward model. The value of this will be explored for applications of APOLLO_NG to more modern sensors than AVHRR.

Online simulations together with the probabilistic approach of retrieving cloud properties have the great advantage that uncertainty assessment is an intrinsic byproduct of each retrieved variable. Consequently, the uncertainty of each observation (i.e., pixel) is estimated from the observations itself. Figure 6 clearly shows the value of such a method. Depending on the purpose of the use of APOLLO_NG results these uncertainties also can be used to confine the applications to observations with high confidence only. This will be especially useful in the application of APOLLO_NG in high resolution case studies (e.g., Klüser et al., 2008) or in the field of aerosol-cloud-interaction research (Klüser et al., 2008; Klüser and Holzer-Popp, 2010).

In this study the methodology of the probabilistic APOLLO_NG scheme has been outlined. The specific cloud detection tests have been motivated from well-known physical principles and standard methodologies of cloud remote sensing. Cloud detection and cloud property evaluation is currently performed for APOLLO_NG by different means and for a range of sensors. Cloud detection will first be cross compared with cloud detection results of the original APOLLO scheme, which has already been evaluated with SYNOP data for AVHRR and for Europe
(Kriebel et al., 2003; Meerkötter et al., 2004). This crosscomparison has already been started and will be described in a subsequent study. Moreover APOLLO_NG will be run with MODIS (Moderate Resolution Imaging Spectroradiometer) and VIIRS observations and will be compared with independent cloud mask results from the MODIS/VIIRS cloud detection (which exploit more channels of these instruments than APOLLO_NG does, see for example Frey et al., 2008). In order to evaluate the cloud property retrievals, the APOLLO_NG results will also be compared to MODIS/VIIRS cloud retrievals. Using external data as a reference also facilitates sensitivity studies, especially in terms of relationships between minimum cloud probability and false alarm rate and cloud detection rate. Moreover the availability of a wide range of channels including those centered at $1.3,1.6,2.2$ and $3.7 \mu \mathrm{m}$ provides the possibility to assess the sensitivity of the retrieved cloud properties to the selection of the absorbing channel. Consequently, the external uncertainties and potential biases for sensors having only one of the useful channels (like AVHRR) can be examined.

\section{Summary and outlook}

Based on the legacy and the physical principles of the wellknown cloud retrieval scheme APOLLO a new scheme called APOLLO_NextGeneration has been developed. By building on the classical principles for cloud detection and cloud property retrieval, these have been implemented in a probabilistic way. This study explained the physical and mathematical principles and approaches used in the formulation of the APOLLO_NG scheme and shows example results with AVHRR. One of the major achievements of the APOLLO_NG scheme is to harmonize the legacy of the APOLLO method for all satellite sensors maintaining the socalled AVHRR heritage channels. So far each sensor had its own APOLLO adaptation (see Kriebel et al., 2003; HolzerPopp et al., 2008; Klüser and Holzer-Popp, 2010). Consequently, a harmonization effort was strongly required for future applications and extended evaluation of cloud products and derived information. As the APOLLO_NG makes use of a probabilistic approach to cloud detection, it thus addresses the need of variable cloud detection conservativeness in a broad range of applications (e.g., Holzer-Popp et al., 2013). Traceability of the origins of the reported cloud probability as well as flexible cloud detection thresholds together with a propagation of information allows for a more useful description of the observed conditions (Fig. 7). New additional interpretations of the cloud detection results such as the Shannon information content for cloud detection moreover feed into the potential to define cloud masks addressing the purpose of the application.

An initial comparison with the traditional APOLLO cloud detection scheme showed that $78-79 \%$ of cloud fraction retrievals from APOLLO_NG fall within $\pm 12.5 \%( \pm 1 / 8)$ of 
APOLLO. This comparison has been done for cloud fraction and for one overpass over Europe in this study. Comparing the resulting numbers with other overpasses in other seasons indicated that these remain fairly constant. Nevertheless, a more rigorous validation of the cloud detection and cloud property retrieval is necessary and will be covered in full detail in a subsequent study.

Introducing the retrieval of cloud droplet (and ice crystal) effective radius into the scheme makes APOLLO_NG also a suitable candidate for aerosol-cloud-interactions research (see Klüser and Holzer-Popp, 2010). APOLLO_NG facilitates the possibility to continue and expand the use of APOLLO in a wide range of applications (e.g., Gesell, 1989; Meerkötter et al., 2004; Holzer-Popp et al., 2008; Klüser and Holzer-Popp, 2010; Qu et al., 2012). All of these applications require a well-understood error characterization as well as clearly documented sensitivities of the APOLLO_NG cloud products. Consequently, a subsequent APOLLO_NG evaluation study will use MODIS and VIIRS data to derive the sensitivity to absorbing channel selection between 1.6 and $3.7 \mu \mathrm{m}$. Moreover, it will cross-examine the cloud property results with the MODIS/VIIRS cloud products at given sensor resolution and geometry.

Acknowledgements. We thank three anonymous reviewers for their constructive comments and valuable suggestions, which helped to improve the manuscript. The development of the APOLLO_NG method was part of the DLR project TIMELINE.

The article processing charges for this open-access publication were covered by a Research

Centre of the Helmholtz Association.

Edited by: A. Sayer

\section{References}

Baum, B. A., Yang, P., Heymsfield, A. J., Bansemer, A., Cole, B. H., Merrelli, A., Schmitt, C., and Wang, C.: Ice cloud singlescattering property models with the full phase matrix at wavelengths from 0.2 to $100 \mu \mathrm{m}$, J. Quant. Spectrosc. Ra., 146, 123139, doi:10.1016/j.jqsrt.2014.02.029, 2014.

Chang, F.-L. and Li, Z.: A new method for detection of cirrus overlapping water clouds and determination of their optical properties, J. Atmos. Sci., 62, 3993-4009, 2005.

Coakley, J. A. and Chylek, P.: The two-stream approximation in radiative transfer: including the angle of incident radiation, J. Atmos. Sci., 32, 409-418, 1975.

Comstock, J. M., d'Entremont, R., DeSlover, D., Mace, G. G., Matrosov, S. Y., McFarlane, S. A., Minnis, P., Mitchell, D., Sassen, K., Shupe, M. D., Turner, D. D., and Wang, Z.: An intercomparison of microphysical retrieval algorithms for uppertropospheric ice clouds, B. Am. Meteorol. Soc., 88, 191-204, 2007.

Frey, R. A., Ackerman, S. A., Liu, Y., Strabala, K. I., Zhang, H., Key, J. R., and Wang, X.: Cloud detection with
MODIS. Part I: Improvements in the MODIS cloud mask for collection 5, J. Atmos. Ocean. Tech., 25, 1057-1072, doi:10.1175/2008JTECHA1052.1, 2008.

Gesell, G.: An algorithm for snow and ice detection using AVHRR data an extension to the APOLLO software package, Int. J. Remote Sens., 10, 897-905, 1989.

Guignard, A., Stubenrauch, C. J., Baran, A. J., and Armante, R.: Bulk microphysical properties of semi-transparent cirrus from AIRS: a six year global climatology and statistical analysis in synergy with geometrical profiling data from CloudSatCALIPSO, Atmos. Chem. Phys., 12, 503-525, doi:10.5194/acp12-503-2012, 2012.

Hirsch, E., Koren, I., Levin, Z., Altaratz, O., and Agassi, E.: On transition-zone water clouds, Atmos. Chem. Phys., 14, 90019012, doi:10.5194/acp-14-9001-2014, 2014.

Holzer-Popp, T., Schroedter-Homscheidt, M., Breitkreuz, H., Martynenko, D., and Klüser, L.: Improvements of synergetic aerosol retrieval for ENVISAT, Atmos. Chem. Phys., 8, 7651-7672, doi:10.5194/acp-8-7651-2008, 2008.

Holzer-Popp, T., de Leeuw, G., Griesfeller, J., Martynenko, D., Klüser, L., Bevan, S., Davies, W., Ducos, F., Deuzé, J. L., Graigner, R. G., Heckel, A., von Hoyningen-Hüne, W., Kolmonen, P., Litvinov, P., North, P., Poulsen, C. A., Ramon, D., Siddans, R., Sogacheva, L., Tanre, D., Thomas, G. E., Vountas, M., Descloitres, J., Griesfeller, J., Kinne, S., Schulz, M., and Pinnock, S.: Aerosol retrieval experiments in the ESA Aerosol_cci project, Atmos. Meas. Tech., 6, 1919-1957, doi:10.5194/amt-61919-2013, 2013.

Kaufman, Y. J. and Nakajima, T.: Effect of Amazon smoke on cloud microphysics and albedo - analysis from satellite imagery, J. Appl. Meteorol., 32, 729-744, 1993.

Klüser, L. and Holzer-Popp, T.: Relationships between mineral dust and cloud properties in the West African Sahel, Atmos. Chem. Phys., 10, 6901-6915, doi:10.5194/acp-10-6901-2010, 2010.

Klüser, L. and Schepanski, K.: Remote sensing of mineral dust over land with MSG infrared channels: A new Bitemporal Mineral Dust Index, Remote Sens. Environ., 113, 1853-1867, doi:10.1016/j.rse.2009.04.012, 2009.

Klüser, L., Rosenfeld, D., Macke, A., and Holzer-Popp, T.: Observations of shallow convective clouds generated by solar heating of dark smoke plumes, Atmos. Chem. Phys., 8, 2833-2840, doi:10.5194/acp-8-2833-2008, 2008.

Kolmogorov, A. N.: Logical basis for information theory and probability theory, IEEE T. Inform. Theory, 14, 662-664, 1968.

Koren, I., Oreopoulos, L., Feingold, G., Remer, L. A., and Altaratz, O.: How small is a small cloud?, Atmos. Chem. Phys., 8, 3855-3864, doi:10.5194/acp-8-3855-2008, 2008.

Kriebel, K. T., Saunders, R. W., and Gesell, G.: Optical properties of clouds derived from fully cloudy AVHRR pixels, Beitr. Phys. Atmosph., 62, 165-171, 1989.

Kriebel, K. T., Gesell, G., Kästner, M., and Mannstein, H.: The cloud analysis tool APOLLO: improvements and validations, Int. J. Remote Sens., 24, 2389-2408, 2003.

MacKay, D. J. C.: Information Theory, Inference and Learning Algorithms, Cambridge University Press, Cambridge, UK, 2004.

Merchant, C. J., Harris, A. R., Maturi, E., and Maccallum, S.: Probabilistic physically based cloud screening of satellite infrared imagery for operational sea surface temperature retrieval, Q. J. Roy. Meteorol. Soc., 131, 2735-2755, doi:10.1256/qj.05.15, 2005. 
Meerkötter, R., König, C., Bissoli, P., Gesell, G., and Mannstein, H.: A 14-year European Cloud Climatology from NOAA/AVHRR data in comparison to surface observations, Geophys. Res. Lett., 31, L15103, doi:10.1029/2004GL020098, 2004.

Nakajima, T. and King, M. D.: Determination of the optical thickness and effective particle radius of clouds from reflected solar radiation measurements - Part I: Theory, J. Atmos. Sci., 47, 1878-1893, 1990.

Oumbe, A., Blanc, P., Ranchin, T., Schroedter-Homscheidt, M., and Wald, L.: A new method for estimating solar energy resource, in: Proceedings of the ISRSE 33, Stresa, Italy, 4-9 May 2009, Joint Research Center, Ispra, Italy, USBKey, paper 773, 2009.

Pavolonis, M. J. and Heidinger, A. K.: Daytime cloud overlap detection from AVHRR and VIIRS, J. Appl. Meteorol., 43, 762-778, 2004.

Qu, Z., Oumbe, A., Blanc, P., Lefevre, M., Wald, L., SchroedterHomscheidt, M., Gesell, G., and Klüser, L.: Assessment of Heliosat-4 surface solar irradiance derived on the basis of SEVIRI-APOLLO cloud products, Proceedings of the 2012 EUMETSAT Meteorological Satellite Conference, Sopot, Poland, 3-7 September 2012, EUMETSAT P. 61, s2-06, 2012.

Rosenfeld, D. and Lensky, I. M.: Satellite-based insights into precipitation formation processes in continental and maritime convective clouds, B. Am. Meteorol. Soc., 79, 2457-2476, 1998.

Rodgers, C.D.: Information content and optimization of high spectral resolution remote measurements, Adv. Space Res., 21, 361367, 1998.
Rodgers, C. D.: Inverse methods for atmospheric sounding, theory and practice, in: Series on Atmospheric, Oceanic and Planetary Physics, vol. 2, World Scientific Publishing, Singapore, 2000.

Saunders, R. W. and Kriebel, K. T.: An improved method for detecting clear sky and cloudy radiances from AVHRR data, Int. J. Remote Sens., 9, 123-150, 1988.

Shannon, C. E. and Weaver, W.: The Mathematical Theory of Communication, University of Illinois Press, 1949.

Shenk, W. E. and Curran, R. J.: The detection of dust storms over land and water with satellite visible and infrared measurements, Mon. Weather Rev., 102, 830-837, 1974.

Stephens, G. L.: Radiation profiles in extended water clouds. II: Parameterization schemes, J. Atmos. Sci., 35, 2123-2132, 1978.

Stephens, G. L., Ackerman, S., and Smith, E. A.: A shortwave parameterization revised to improve cloud absorption, J. Atmos. Sci., 41, 687-690, 1984.

Yang, P., Wei, H., Huang, H.-L., Baum, B. A., Hu, Y. X., Kattawar, G. W., Mishchenko, M. I., and Fu, Q.: Scattering and absorption property database for nonspherical ice particles in the near- through far-infrared spectral region, Appl. Optics, 44, 5512-5523, 2005.

Warren, S. G.: Optical constants of ice from the ultraviolet to the microwave, Appl. Optics, 23, 1206-1225, 1984. 